\title{
Perfil respiratório dos trabalhadores
da indústria moveleira do município
de Coronel Freitas-SC* de Coronel Freitas-SC*
}

Respiratory profile of the furniture

industry workers in the city of

Coronel Freitas -SC*

FisiSenectus. Unochapecó Ano 1, n. 1 - Jan./Jun. 2013 p. $43-50$

*Trabalho de conclusão de curso apresentada à Unochapecó como parte dos requisitos para obtenção do grau de Bacharel em Fisioterapia.

\section{Indiamara de Oliveira Flores Dal Magro Silvani}

Fisioterapeuta, mestre em Engenharia Biomédica, Unochapecó.

\section{Eliane Grasel}

Fisioterapeuta, Unochapecó.

\section{Suelen Pansera}

Fisioterapeuta, Unochapecó.

\section{Resumo}

Introdução: nas indústrias que utilizam a madeira como matéria-prima, várias partículas estão suspensas no ar e o ambiente é rico em substâncias químicas e partículas orgânicas. 0 trato respiratório está diretamente em contato com o meio ambiente e acredita-se que a inalação de aerodispersoides possa causar diferentes tipos de respostas ou dano local no organismo. Durante o processamento da madeira nos seus vários tipos de emprego, finas partículas são produzidas. 0 tamanho das partículas de poeira tem entre 1,3 a 33 micrômetros $(\mu \mathrm{m})$. A reação pela poeira da madeira é frequentemente encontrada em carpinteiros, marceneiros, serradores e polidores. Objetivo: traçar o perfil respiratório por meio da espirometria dos trabalhadores expostos à poeira de madeira da indústria moveleira do município de Coronel Freitas-SC. Materiais e métodos: foi realizado um estudo com 120 testes espirométricos dos trabalhadores das indústrias de móveis do município, atendidos durante o período de um ano, entre 2006 e 2007, vinculados a várias funções com exposição à poeira da madeira. Resultados: os testes demonstram que apenas 1,67\% está com alteração na Capacidade Vital, Capacidade Vital Forçada, Volume Expiratório Forçado no primeiro segundo e a Ventilação Voluntária Máxima. A exposição à poeira da madeira pode contribuir para problemas respiratórios, mas a prevalência da normalidade nas espirometrias está presente na amostra estudada, com 6,66\% dos indivíduos com distúrbios ventilatórios. Porém, ressalta-se a necessidade de um trabalho preventivo nas indústrias de móveis, visando à redução da exposição do trabalhador à poeira, diminuindo assim o risco de futuras patologias.

\section{Palavras-chave}

Poeira. Sistema respiratório. Espirometria.

\section{Fisiß̌enectus}




\begin{abstract}
Introduction: in the industries that use the wood as raw material, several particles are suspended in the air and the atmosphere is rich in chemical substances and organic particles. The respiratory system is directly in contact with the environment and it is believed that the aerodispersive inhalation can cause different types of reaction or local damage in the body. During the processing of the wood in its several uses, fine particles are produced. The size of the dust particles has among 1,3 to 33 micrometers $(\mu \mathrm{m})$. The reaction of the dust wood is frequently found in carpenters, cabinetmaker, sawyers and polishers. Materials and methods: a study was accomplished with the industries workers of pieces in Coronel Freitas city 120 spirometric tests, assisted during the period of one year, between 2006 and 2007, linked to several functions exposed to the dust of the wood. Results: demonstarm that $1,67 \%$ are just with alterations in the Vital Capacity, Vital Capacity Forced, Expiratory Volume Forced in the first second and to Maximum Voluntary Ventilation. The exhibition to the dust of the wood, second several authors, it can contribute to breathing problems, but the prevalency of the normality in the spirometry is present in the studied sample, with $6,66 \%$ of the individuals with ventilatory disturbances. Even so, it stood out the need of a preventive work in the industries of pieces of furniture, seeking to the reduction of the orker exposure to the dust, decreasing like this the risk of future pathologies.
\end{abstract}

\title{
Keywords
}

Exercise. Hypertension. Blood pressure.

\section{Introdução}

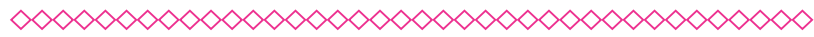

A prevenção de patologias relacionadas ao local de trabalho tem se fortalecido ao longo dos anos e impulsionadas pelo interesse na qualidade de vida dos trabalhadores, buscando formas de minimizar as doenças ocupacionais e, consequentemente, prevenindo as incapacidades decorrentes dessas doenças. A visão de atenção integral à saúde no trabalho impulsiona um novo campo de atuação dos profissionais da saúde, em que é preciso entender o processo saúde-doença, buscando identificar e avaliar os riscos à saúde presentes nos locais de trabalho, sugerindo mudanças no estilo de vida destes trabalhadores.

Nas indústrias, várias partículas estão suspensas no ar, oriundas dos processos de fabricação em geral, sendo um ambiente rico em substâncias químicas. Nas industrias moveleiras também há um grande número de partículas orgânicas vindas da utilização da madeira nos processos industriais. ${ }^{1}$

Os danos à saúde humana advêm não somente de poeiras orgânicas, mais de uma gama de "agentes ativos", produzidos ou contidos nesse material particulado orgânico com potencial de produzir efeitos biológicos. As poeiras orgânicas podem ocasionar patologias como: asma alérgica, síndrome torácica por poeira orgânica e pneumonite por hipersensibilidade ${ }^{2}$.

O homem precisa estar saudável para poder desenvolver bem sua tarefa no trabalho. Para isso, as condições ambientais e do próprio posto de trabalho precisam estar adequadas. Se as doenças ocupacionais estão presentes, a produtividade não será a mesma e a qualidade de vida estará prejudicada. Dentro desta visão, a segurança e a saúde ocupacional têm sido cada vez mais abordadas, baseadas nas exigências da sociedade e apoio legal, como as Normas Regulamentadoras do Ministério do Trabalho, que visam garantir a segurança e saúde do trabalhador no ambiente em que está inserido.

Por isso, buscamos neste texto traçar o perfil respiratório por intermédio da espirometria dos trabalhadores expostos à poeira de madeira da indústria moveleira do município de Coronel Freitas-SC. Procuramos, também, identificar se existem alterações dos parâmetros espirométricos, observando se o perfil traçado está dentro das normalidades, encontrar e classificar a presença de distúrbios ventilatórios dos indivíduos, observar se ocorre relação entre tempo de exposição e alterações nos valores espirométricos e traçar possíveis metas de prevenção para melhorar alterações, caso encontradas. 


\section{Materiais e métodos}

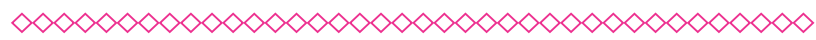

Caracteriza-se por um estudo quantitativo tendo um caráter exploratório - descritivo. $\mathrm{Na}$ pesquisa exploratória, o pesquisador parte de uma hipótese e aprofunda seu estudo nos limites de uma realidade específica, buscando antecedentes e maiores conhecimentos para, em seguida, planejar uma pesquisa descritiva ou do tipo experimental. Um estudo exploratório pode servir para levantar problemas de pesquisa ${ }^{3}$.

Foi realizado um estudo nos testes espirométricos realizados por trabalhadores da indústria de móveis de madeira do município de Coronel Freitas-SC. A amostra foi composta por 120 exames de espirometria, representando $12 \%$ dos trabalhadores da indústria de móveis de madeira. Os critérios de inclusão: gênero masculino; idade acima de 20 anos; tempo de exposição à poeira de madeira acima de quatro anos; ter realizado pelo menos um teste espirométrico nos anos de 2006 ou 2007.

A seleção dos testes espirométricos foi realizada aleatoriamente. Foi realizado o estudo incluindo 120 testes espirométricos dos trabaIhadores atendidos durante o período de um ano, entre 2006 e 2007, provenientes da demanda ambulatorial do estabelecimento Alphamed - Medicina do Trabalho LTDA., no município de Coronel Freitas-SC. Os exames foram realizados neste estabelecimento e o aparelho de espirometria utilizado nas provas de função pulmonar foi o da marca Spirom 2, sendo este volumétrico, juntamente com o Espiromatic 32,4.

Levantaram-se as informações relacionadas ao trabalhador e a sua exposição no local de trabalho: função desenvolvida, tempo de exposição em anos dos trabalhadores à poeira da madeira, idade em anos, peso em quilogramas, altura em centímetros, Índice de Massa Corporal (IMC) dos trabalhadores, em quilogramas por metro ao quadrado $\left(\mathrm{Kg} / \mathrm{m}^{2}\right)$.

Para observar a presença ou não de alterações dos parâmetros espirométricos, os dados com maior relevância para a pesquisa analisados foram: Capacidade Vital (CV), Capacidade Vital Forçada (CVF), Volume Expiratório Forçado no Primeiro Segundo $\left(\mathrm{VEF}_{1}\right)$, Ventilação Voluntária Máxima (VVM).
Para análise dos resultados, os valores foram apresentados em forma de tabelas e distribuídos estatisticamente em média, desvio padrão, mediana, máxima e mínima. Os valores foram obtidos por meio de análise estatística simples pelo programa Microsoft Excel 2003. Os valores obtidos nos testes espirométricos foram comparados com valores presentes na literatura atual e parâmetros definidos no I Consenso Brasileiro sobre Espirometria de 1996.

\section{Resultados}

$\infty<\infty<\infty<\infty<\infty<\infty<\infty<\infty<\infty<\infty<\infty<\infty<\infty<\infty<\infty$

A partir dos dados obtidos, constatou-se que os 120 trabalhadores estão em contado direto com a poeira da madeira. Destes, $51,67 \%$ são operadores de máquina, $24,17 \%$ são auxiliares de produção, 8,33\% são operadores de lixadeira, 5,01\% são auxiliares de montagem de móveis, 3,33\% são auxiliares de marceneiro e os demais $8,33 \%$ possuem outras funções, como plainador, lixador, marceneiro, entre outras ocupações.

A tabela 1 apresenta dados sobre o tempo de exposição em anos dos trabalhadores à poeira da madeira, e neste evidencia-se que 41,67 trabaIham entre 10 a 12 anos nessa função.

A massa corporal dos trabalhadores foi mensurada em quilogramas ( $\mathrm{kg}$ ), em que $5 \%$ apresentaram um peso entre 45 a $55 \mathrm{~kg}$; 28,34\% entre 56 a 65 kg; 30,83\% entre 66 a 75 kg; 25,83\% entre 76 a $85 \mathrm{~kg}$; e $10 \%$ apresentaram um peso maior ou igual a $85 \mathrm{~kg}$. Os valores obtidos tiveram uma média de $71,57 \mathrm{~kg} \pm 10,95$ e uma mediana de $70 \mathrm{~kg}$.

$\mathrm{Na}$ pesquisa, $6,67 \%$ dos trabalhadores possuem altura entre 150 a $160 \mathrm{~cm}, 47,60 \%$ com estatura entre 161 a $170 \mathrm{~cm}, 42,50 \%$ com 171 a $180 \mathrm{~cm}$ e $3,33 \%$ possuem altura maior ou igual a $181 \mathrm{~cm}$. Apresentando uma média de $170 \mathrm{~cm} \pm 6,48$; uma mediana de $187 \mathrm{~cm}$, o valor máximo encontrado de $187 \mathrm{~cm}$ e o valor mínimo de $152 \mathrm{~cm}$.

No presente estudo $71 \%$ dos trabalhadores apresentaram IMC dentro das normalidades, entre 18,5 a $25 \mathrm{~kg} / \mathrm{m}^{2} ; 39,17 \%$ da amostra apresentaram valores acima do normal. Os valores obtidos tiveram uma média de $24,44 \mathrm{~kg} / \mathrm{m}^{2} \pm 3,17$ uma mediana de $24,11 \mathrm{~kg} / \mathrm{m}^{2}$. 
Já no que diz respeito sobre as capacidades pulmonares, os valores de referência utilizados pelo software do espirômetro foram os preconizados por Pereira et al. (1992), levando em consideração as particularidades de cada indivíduo (sexo, altura, peso e idade) $)^{5}$.

Os valores encontrados para a capacidade vital e capacidade vital forçada alcançaram uma média de 4,63 $\pm 0,76$; uma mediana de 4,54, apresentando 2,94 como valor mínimo e 6,52 sendo valor máximo.

A tabela 2 apresenta dados sobre a capacidade vital e capacidade vital forçada foram mensuradas em litros, onde $98,33 \%$ obtiveram os valores dentro e acima da normalidade e $1,67 \%$ apresentou alteração nesse item do teste espirométrico.

0 Volume Expiratório Forçado no Primeiro Segundo $\left(\mathrm{VEF}_{1}\right)$ apresentou $98,33 \%$ dos testes espirométricos dentro e acima dos valores normais e $1,67 \%$ com valores abaixo da normalidade. Com uma média de 4,63 $\pm 6,92$; uma mediana de 4,00; 2,18 e 79,5 representam respectivamente valores máximos e mínimos, destacados na tabela 3.

A tabela 4 destaca resultados sobre a média da Ventilação Voluntária Máxima (VVM) foi de 147,43 $\pm 24,10$, uma mediana de 147,43 ; onde 80,6 foi o valor mínimo encontrado e 220 o valor máximo encontrado. Em resumo, 98,33\% dos testes espirométricos apresentaram valores dentro e acima da normalidade e $1,67 \%$ valores abaixo da normalidade.

\section{Discussão}

$\infty \times \infty \times \infty \times \infty \times \infty \times \infty \times \infty \times \infty \times \infty \times \infty \times \infty \times \infty \times \infty \infty$

Nas espirometrias que apresentaram alterações, observou-se uma média de 8,57 anos de exposição à poeira da madeira. Já os testes espirométricos de trabalhadores expostos à poeira da madeira por mais tempo (10 a 13 anos) não foram encontradas alterações. Com isso, não é possível afirmar que o tempo prolongado de exposição cause danos à saúde respiratória do trabalhador.

Em estudo realizado sobre os riscos respiratórios desencadeados pela poeira de madeira em indústria de móveis e madeira na cidade de São PauIo, a média de idade encontrada foi de 31,6 anos entre os trabalhadores do sexo masculino. Este estudo vem ao encontro dos atuais resultados encontrados nesta pesquisa.

Constatou-se que os trabalhadores estão com a massa corporal dentro dos limites considerados normais, representando $59,17 \%$. Com isso, os dados obtidos no estudo indicam que 33,33\% dos trabalhadores apresentam sobrepeso, não significando obesidade, porém sendo um agravante para complicações futuras de saúde.

Esses dados põem em evidência as transformações nos estilos de vida e no mundo do trabalho, sugerindo a emergência de hábitos que seriam, segundo alguns estudos, resultantes do ajuste às novas formas de consumo e aos novos padrões tecnológicos, além dos processos de urbanização e migração. ${ }^{8,9}$

No que se referem às espirometrias, diversas equações para valores de referência foram publicadas nas últimas décadas. Os valores esperados para indivíduos com uma dada combinação de idade e estatura podem diferir consideravelmente. Tais variações podem ser explicadas pelos critérios de seleção das populações "normais", pelos equipamentos usados, pelas técnicas de medida, pela variabilidade biológica das populações e pelos modelos estatísticos utilizados na análise dos dados $^{10}$.

Com isso, alguns autores criaram algumas fórmulas para obter valores de referência de cada indivíduo. Como essas contas iriam despender de muito tempo, os softwares dos espirômetros realizam essa equação, apresentando os limites máximos e mínimos que o paciente pode apresentar. As fórmulas utilizadas para obtenção dos resultando das capacidades pulmonares para este estudo foram as preconizadas por estudos anteriores ${ }^{4}$.

A capacidade vital - forçada ou lenta - origina-se na capacidade pulmonar total (CPT) e termina no volume residual (VR). No estudo realizado, 98,33\% dos testes espirométricos apresentaram capacidade vital (CV) e capacidade vital forçada (CVF) com valores dentro e acima dos limites normais e $1,67 \%$ dos valores abaixo dos limites normais ${ }^{11}$.

No estudo, o Volume Expiratório Forçado no primeiro segundo apresentou $98,33 \%$ dos testes 
espirométricos dentro e acima dos valores normais e $1,67 \%$ valores abaixo da normalidade.

O VEF $F_{1}$ contudo, é um teste relativamente insensível para avaliar a doença pulmonar na fase inicial e pode inclusive ser normal, mesmo quando há doença pulmonar clinicamente significativa. Dessa forma, os testes de função pulmonar tornaram-se um instrumento de valor não só no monitoramento da perda da função pulmonar ao longo do tempo como também na monitorização da melhora da função pulmonar em resposta às várias estratégias terapêuticas empregadas. ${ }^{12}$

Os trabalhadores na indústria de móveis dinamarquesa que trabalham com o pinho têm um aumento nas queixas de uma respiração difícil. A exposição à poeira de madeira de pinho mostrou uma diminuição no volume expiratório forçado em 1s $\left(\mathrm{FEV}_{1}\right)$ em pacientes com sintomas de asma ${ }^{13}$.

A Ventilação Voluntária Máxima é o maior volume de ar que o indivíduo pode mobilizar em um minuto com esforço voluntário máximo. 0 teste dá uma visão global inespecífica da função ventilatória. No passado, o teste era chamado de Capacidade Respiratória Máxima (CRM). A manobra do VVM é geralmente feita por um período de dez a 15 segundos e o volume neste período de tempo é então extrapolado para o valor de um minuto. As unidades usadas são L/min. VVM pode ser medida em qualquer espirômetro. Sistemas modernos registram o volume acumulado que é mobilizado durante o tempo de medida ${ }^{4,5}$.

Levando em consideração a particularidade de cada indivíduo, o perfil do trabalhador da indústria moveleira do município de Coronel Freitas, exposto à poeira da madeira, apresenta as seguintes características, considerando a média: 34,4 anos; 187 centímetros de altura, $71,565 \mathrm{~kg}, 8,59$ anos de exposição à poeira da madeira, IMC $24,63 \mathrm{~kg} / \mathrm{m}^{2}$, capacidade vital (CV) 4,63L; capacidade vital forçada (CVF) 4,63L; Volume Expiratório Forçado no Primeiro Segundo $\left(\operatorname{VEF}_{1}\right) 4,63 \mathrm{~L}$, Ventilação Voluntária Máxima (VVM) 147,43 L/min.

$\mathrm{Na}$ conclusão das espirometrias, os resultados mostram que a maioria dos testes são considerados dentro da normalidade, contemplando 93,34\% dos exames. 0 distúrbio mais frequente encontrado foi o ventilatório obstrutivo, com sete expos- tos, representando 5,83\% da amostra. 0 distúrbio ventilatório restritivo esteve presente em apenas um trabalhador, significando $0,83 \%$. Os distúrbios ventilatórios misto e inespecífico não foram encontrados na amostra estudada.

As medidas espirométricas devem ser feitas próximas ou no local de trabalho para evitar um lapso de tempo longo entre a exposição ocupacional e a mudança funcional. Este pode ter sido um dos motivos da prevalência da normalidade nos testes, já que estes foram realizados longe do local de trabalho ${ }^{4}$. A prevalência de resultados normais das espirometrias, 93,34\%, está de acordo com outros trabalhos realizados na área de pneumopatias ocupacionais. A espirometria não parece ser um exame com boa sensibilidade para detectar alterações precoces de comprometimento respiratório.

Em análise de espirometrias em trabalhadores de indústrias moveleiras, de ambos os sexos, na faixa etária dos 20-65 anos, com média de idade de $33,33( \pm 9,10)$ anos. Dos participantes, 90,2\% $(n=46)$ dos indivíduos apresentaram espirometria normal, 7,8\% ( $n=4)$ apresentaram Distúrbio Ventilatório Obstrutivo Leve (DVOL) e apenas 1,9\% $(n=1)$ apresentou Distúrbio Ventilatório Restritivo Leve (DVRL) ${ }^{14}$.

Rosa et al. ${ }^{6}$ realizaram uma avaliação dos riscos respiratórios desencadeados por poeira de madeira em indústria de móveis e madeira na cidade de São Paulo. Realizaram a avaliação da função pulmonar dos trabalhadores do setor de marcenaria, em um total de 58 espirometrias simples. Três apresentaram restrição leve de vias aéreas, não havendo prova com obstrução de vias aéreas. Duas provas tiveram redução de FEF-25-75\% da CVF .

Portanto, mesmo com a prevalência da normalidade nas espirometrias da amostra estudada, registra-se que as consequências à exposição à poeira da madeira poderiam ser perfeitamente evitáveis se fossem objeto de atenção de programas de prevenção. As exposições resultantes de ambientes ocupacionais podem ser reduzidas ou eliminadas com ações mais efetivas de controle de poeiras nos ambientes de trabalho e ações de vigilância em saúde do trabalhador. Sabe-se que cuidados e uso de EPIs (Equipamentos de Proteção Individuais) são muitas vezes negligenciados. 
No setor moveleiro, estima-se que apenas $13 \%$ a $15 \%$ das plantas industriais possuam sistemas de exaustão central para captação de pó de serra, plaina e lixa (estimativa para polos). Empresas pequenas não possuem exaustores e suas emissões são liberadas, dispensadas pelo vento, na cidade ou área industrial. Outros particulados emitidos são as resinas e tintas (originadas nas operações de pintura e envernizamento); e a fumaça particulada (com resíduos químicos) de queima não controlada ${ }^{15}$.

O estado de Santa Catarina apresentava vários polos moveleiros consolidados e potenciais no País (São Bento do Sul, Rio Negrinho, Coronel Freitas, Pinhalzinho e São Lourenço do Oeste $\left.{ }^{16}\right)$. 0 fato da região oeste de Santa Catarina ser um dos maiores exportadores de móveis do País favorece e, consequentemente, eleva o número de expostos à poeira da madeira.

As características de um ambiente de trabalho refletem, de maneira expressiva, as qualidades do trabalhador. Um local de trabalho deve ser sadio e agradável, que proporcione o máximo de proteção, sendo o resultado de fatores materiais ou subjetivos, e devem prevenir acidentes, doenças ocupacionais, além de proporcionar melhor relacionamento entre a empresa e o empregado ${ }^{17}$.

Portanto, algumas medidas de prevenção podem ser tomadas, como realizar um estudo ergonômico do ambiente de trabalho, proporcionar palestras e cursos de orientação sobre segurança no trabalho, realizar revezamento das atividades para que o funcionário não esteja sempre em contato com a poeira da madeira e outros resíduos químicos. A mensuração da concentração de poeira de madeira nas indústrias deve ser avaliada, pois é uma forma de conhecer qual a concentração que está sendo inalada. A utilização dos EPIs são de suma importância e não podem ser negligenciados, pois é uma garantia de segurança à saúde do trabalhador.

Podem ser implantados sistemas de ventilação exaustora com coletores de poeiras nos locais de polimento, da serra circular, fresas e outras máquinas; umectação do ambiente, quando partículas forem maiores de $5 \mu \mathrm{m}$ (cinco micrômetros) de diâmetro; o controle do pó originado do polimento mecânico recomendado entre 1 a $10 \mathrm{mg} / \mathrm{m}^{3}$ de partícula no ambiente ${ }^{18}$.
A partir de toda importância e necessidade da prevenção de patologias nos postos de trabalho, observa-se um campo de atuação para o fisioterapeuta, em que pode contribuir com propostas educativas de conscientização tanto para o trabaIhador quanto para o empregador, visando à necessidade e os benefícios da utilização dos equipamentos de proteção individual. Pode, também, atuar na área ergonômica do local de trabalho, além do incentivo a realização da mensuração da concentração da poeira.

\section{Conclusão}

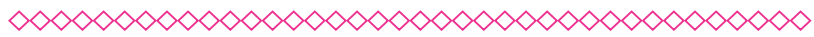

Fica um questionamento se a espirometria é um exame de fácil detecção de alterações pulmonares ocupacionais relacionadas à exposição à poeira da madeira, já que a maioria dos testes espirométricos foram considerados dentro da normalidade. Desta forma, a espirometria pode não ser o suficiente no que diz respeito à detecção precoce de alterações nos volumes e capacidades pulmonares, retardando, deste modo, o diagnóstico e tratamento.

Porém, ressalta-se a necessidade de um trabaIho com um foco preventivo nas indústrias de móveis, com a inserção do fisioterapeuta neste local, visando à redução da exposição do trabalhador à poeira, diminuindo, assim, o risco de futuras patologias.

A ação da poeira da madeira no organismo humano é pouco conhecida, por isso deve ser investigada e pesquisada com mais profundidade. A preocupação com os efeitos da poeira na saúde no homem é explorada de uma forma limitada, portanto, as pesquisas devem ser incentivadas.

\section{Referências}

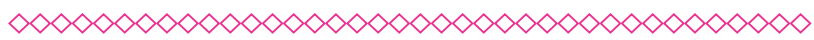

1. Guimarães, PA. Avaliação funcional pulmonar em picadores de madeira de indústria de papel e celulose. In. Serviço Social da Indústria do Papel, Papelão e Cortiça do Estado de São Paulo. 
2. Mendes, R. Patologia do trabalho. 2 ed. Atual e ampl. São Paulo: Atheneu, 2005.

3. Omasi, NGS; Yamamoto, RM. Metodologia da pesquisa em saúde: fundamentos essenciais. Curitiba: Autores, 1999.

4. Pereira, CAC. (Coord). Consenso brasileiro sobre espirometria. Jornal de Pneumologia, São Paulo: 1996. v. 22, p.105-164.

5.Torloni M, Vieira VA. Manual de proteção respiratória. São Paulo: 2003.

6. Rosa EA, Brito MEL, Almeida AMMP, Baroni TM. Avaliação dos riscos respiratórios desencadeados por poeira de madeira em indústria de móveis e madeira na cidade de São Paulo. Rede especial - revista do projeto de cooperação técnica BrasilItália, Brasil, ano III, p. 127-44, 1998.

7. Drumond SC. Estudo dos valores de referência de parâmetros espirométricos em crianças e adolescentes com diferentes índices de massa corporal. 2006. 93 p. Dissertação (Mestrado em Saúde da Criança e do Adolescente) Universidade Federal de Minas Gerais: Faculdade de Medicina; Belo Horizonte, 2006.

8. Sampaio LR, Figueirdo VC. Correlação entre o índice de massa corporal e os indicadores antropométricos de distribuição de gordura corporal em adultos e idosos. Revista de Nutrição: 2005. v.18, p. 53-6.

9. Cabrera MAS, Wajngarten M, Gebara OCE, Diament J. Relação do índice de massa corporal, da relação cintura-quadril e da circunferência abdominal com a mortalidade em mulheres idosas: seguimento de 5 anos. Cadernos de saúde pública, Rio de Janeiro: 2005.v.21, p. 767-775.

10. Viana SV, Costa MC, Loureiro S, Veloso IS. Ocorrência de sobrepeso e outros fatores de risco cardiovascular em trabalhadores atendidos em um ambulatório de Nutrição do Centro de Estudos de Saúde do Trabalhador, Salvador-BA. Revista Brasileira de Saúde Ocupacional, São Paulo: 2006.v. 31,p. 19-26.
11. Duarte AAO, Pereira CAC, Rodrigues SCS Validação de novos valores previstos brasileiros para a espirometria forçada na raça branca e comparação com os valores previstos obtidos por outras equações de referência. J. Brás. Pneumol. Asa Sul-Brasília/DF: 2007.v. 33, ed.5, p. 527-535,.

12. Andrade EF, Fonseca DLO, Silva FAA, Barreto SSM. Avaliação evolutiva da espirometria na fibrose cística. J Pneumol. 2001.v. 27,p.130-136 [acesso em 29 mai 2008]. Disponível em: http:// www.scielo.br/pdf/jpneu/v27n3/9224.pdf.

13. Schlunssen V, Vinzents PS, Mikkelsen AB, Schaumburg I. Wood dust exposure in the danish furniture industry using conventional and passive monitors. Ann. occup. Hyg: 2001. v. 45,p. 157-164.

14. Públio AZ. Avaliação de trabalhadores das indústrias moveleiras de Votuporanga-sp, aspectos respiratórios clínicos e funcionais. 2002. 60 p. Mestrado (Mestrado em Fisioterapia) Centro Universitário do Triângulo, 2002.

15. Bertuncello JMZ, Rocha N. Gestão organizacional e desenvolvimento regional no setor moveleiro na região do município de Coronel Freitas-SC. 2007. 139 p. Monografia (Especialização em Formação para o Magistério Superior) - Universidade Comunitária Regional de Chapecó, 2007.

16. Valença ACV, Pamplona LMP, Souto SW. Os novos desafios para a indústria moveleira no Brasil. BNDES Setorial, Rio de Janeiro:2002, p. 83-96.

17. Fiedler NC, Venturolli F, Minetti LJ. Análise de fatores ambientais em marcenarias no Distrito Federal. Rev. Bras. Eng. Agríc. Ambiental, Campina Grande: 2006.v.10,p.679-685.

18. Ali SA. Dermatoses ocupacionais. In: Ferreira JM. Saúde no Trabalho: temas básicos para o profissional que cuida da saúde dos trabalhadores. São Paulo: Roca, 2000. cap. 8, p. 176-226. 


\section{Anexos}

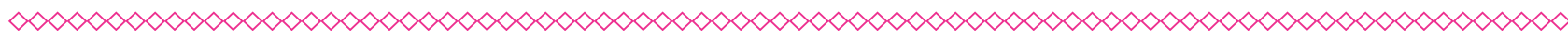

Tabela 1 - Tempo de exposição em anos dos trabalhadores à poeira da madeira

\begin{tabular}{ccc} 
Tempo de exposição & Frequência & Frequência relativa (\%) \\
4 a 6 anos & 28 & 23,33 \\
7 a 9 anos & 40 & 33,33 \\
10 a 12 anos & 50 & 41,67 \\
$\geq 13$ anos & 2 & 1,67 \\
\hline TOTAL & $\mathbf{1 2 0}$ & $\mathbf{1 0 0}$
\end{tabular}

Fonte: elaboração das autoras.

(clique para voltar ao texto)

Tabela 2 - Capacidade Vital (CV) e Capacidade Vital Forçada (CVF), em litros

\begin{tabular}{ccc} 
Valores da CV & Frequência & Frequência relativa (\%) \\
2,94 a 3,94 & 17 & 14,17 \\
3,94 a 4,94 & 61 & 50,83 \\
4,94 a 5,94 & 35 & 29,17 \\
5,94 a 6,52 & 7 & 5,83 \\
\hline TOTAL & $\mathbf{1 2 0}$ & $\mathbf{1 0 0}$ \\
\hline
\end{tabular}

Fonte: elaboração das autoras.

(clique para voltar ao texto)

Tabela 3 - Volume Expiratório Forçado no Primeiro Segundo $\left(\mathrm{VEF}_{1}\right)$, em litros

\begin{tabular}{ccc} 
Valores do VEF1 & Frequência & Frequência relativa (\%) \\
2,18 a 3,18 & 8 & 6,67 \\
3,18 a 4,18 & 61 & 50,83 \\
4,18 a 5,18 & 34 & 28,33 \\
5,18 a 5,94 & 15 & 12,5 \\
$\geq 5,95$ & 2 & 1,67 \\
\hline TOTAL & $\mathbf{1 2 0}$ & $\mathbf{1 0 0}$ \\
\hline
\end{tabular}

Fonte: elaboração das autoras.

(clique para voltar ao texto)

Tabela 4 - Ventilação Voluntária Máxima (VVM), em litros por minuto, de acordo com as características individuais

\begin{tabular}{ccc} 
VVM & Frequência & Frequência relativa (\%) \\
Acima dos valores normais & 76 & 63,33 \\
Dentro dos limites da normalidade & 42 & 35,00 \\
Abaixo dos valores normais & 2 & 1,67 \\
\hline TOTAL & $\mathbf{1 2 0}$ & $\mathbf{1 0 0}$ \\
\hline
\end{tabular}

Fonte: elaboração das autoras.

(clique para voltar ao texto) 